\title{
Perineuronal Nets Regulate the Inhibitory Perisomatic Input onto Parvalbumin Interneurons and $\gamma$ Activity in the Prefrontal Cortex
}

\author{
${ }^{\circledR}$ Hector Carceller, ${ }^{1}{ }^{\circledR}$ Ramon Guirado, ${ }^{1}$ Edna Ripolles-Campos, ${ }^{1}{ }^{\circledR}$ Vicent Teruel-Marti, ${ }^{2}$ and ${ }^{\circledR}$ Juan Nacher ${ }^{1,3,4}$ \\ ${ }^{1}$ Department of Cell Biology, Interdisciplinary Research Structure for Biotechnology and Biomedicine (BIOTECMED), University of Valencia, \\ Burjassot, 46100, Spain, ${ }^{2}$ Neuronal Circuits Laboratory, Department of Anatomy and Human Embryology, University of Valencia, Valencia, 46010, \\ Spain, ${ }^{3}$ Spanish National Network for Research in Mental Health, CIBERSAM, Madrid, 28029, Spain, and ${ }^{4}$ Fundación Investigación Hospital Clínico \\ de Valencia, INCLIVA, Valencia 46010, Spain
}

Parvalbumin-expressing $(\mathrm{PV}+)$ interneurons play a key role in the maturation and synchronization of cortical circuitry and alterations in these inhibitory neurons, especially in the medial prefrontal cortex (mPFC), have been found in different psychiatric disorders. The formation of perineuronal nets (PNNs) around many of these interneurons at the end of the critical periods reduces their plasticity and sets their connectivity. Consequently, the presence of PNNs must have an important impact on the synaptic input and the physiology of PV + cells. In the present study, we have found that in adult male mice, prefrontocortical PV + cells surrounded by PNNs show higher density of perisomatic excitatory and inhibitory puncta, longer axonal initial segments (AISs), and higher PV expression when compared with PV + cells lacking PNNs. In order to better understand the impact of PNNs on the connectivity and physiology of PV + interneurons in the mPFC, we have digested enzymatically these structures and have found a decrease in the density of inhibitory puncta on their perisomatic region but not on the $\mathrm{PV}+$ perisomatic puncta on pyramidal neurons. Moreover, extracellular recordings show that the digestion of PNNs induces a decrease in $\gamma$ activity, an oscillation dependent on PV + cells, in the mPFC of anesthetized mice. Our results suggest that the presence of PNNs enwrapping PV + cells regulates their inhibitory input and has a potent influence on their activity. These results may be relevant for psychiatric research, given the alterations in PNNs, PV+ interneurons and their physiology described in different mental disorders.

Key words: basket cell; $\gamma$ oscillation; interneuron; perineuronal net; prefrontal cortex

Significance Statement

Parvalbumin-expressing $(\mathrm{PV}+)$ interneurons are surrounded by specializations of the extracellular matrix, the perineuronal nets (PNNs). PNNs regulate the development and plasticity of PV+ cells and, consequently, their presence must influence their synaptic input and physiology. We have found, in the adult prefrontal cortex (PFC), substantial differences in the structure and connectivity of PV+ interneurons depending on the presence of PNNs. The depletion of PNNs from the PFC has also a potent effect on the connectivity of $\mathrm{PV}+$ cells and on neural oscillations that depend on these cells. These findings are relevant to understand the role of PNNs in the adult brain and in certain psychiatric disorders in which alterations in PNNs and PV+ interneurons have been described.

Received Feb. 6, 2020; revised Apr. 8, 2020; accepted May 4, 2020

Author contributions: V.T.-M. and J.N. designed research; H.C., R.G., E.R.-C., and V.T.-M. performed research; H.C., R.G., E.R.-C., and V.T.-M. analyzed data; H.C., R.G., V.T.-M., and J.N. wrote the paper.

This work was supported by the Spanish Ministry of Economy and Competitiveness Grants SAF2015-68436R and RTI2018-098269-B-I00 (to J.N.) and BFU2016-77691-C2-2-P (to V.T.-M.). H.C. had a predoctoral fellowship from the Spanish Ministry of Science, Innovation and Universities (FPU15/01233). R.G. had a postdoctoral fellowship "Juan de la Cierva" from the Spanish Ministry of Science, Innovation and Universities (IJCI-2016-27758).

The authors declare no competing financial interests.

Correspondence should be addressed to Juan Nacher at nacher@uv.es.

https://doi.org/10.1523/JNEUROSCI.0291-20.2020

Copyright $\odot 2020$ the authors

\section{Introduction}

Interneurons constitute a minor population in the neocortex, although their role in controlling and synchronizing cortical circuits is crucial (Tremblay et al., 2016). Although neocortical interneurons are highly heterogeneous, parvalbumin-expressing $(\mathrm{PV}+)$ cells constitute the largest interneuronal population in the mammalian cortex; these PV + interneurons can be further classified in fast-spiking basket cells and chandelier cells (Tremblay et al., 2016).

$\mathrm{PV}+$ basket interneurons constitute the main source of perisomatic inhibition both onto excitatory and inhibitory neurons 
(Freund and Katona, 2007; Pfeffer et al., 2013), along with perisomatic synapses from cholecystokinin $\left(\mathrm{CCK}^{+}\right)$expressing large basket cells, which express the cannabinoid receptor 1 (CB1r; Freund and Katona, 2007). The PV + basket cells show a fast and non-adapting firing pattern, providing a synchronized modulation of cortical activity through the regulation of $\gamma$ oscillations (Kubota, 2014). These interneurons are involved in cognitive tasks and, interestingly, the expression level of PV can be used to classify them in different subpopulations regarding their activity and connectivity patterns (Donato et al., 2013, 2015).

A trait of most neocortical PV + cells is the coverage of their soma and proximal dendrites by aggregated lattice-like structures of extracellular matrix called perineuronal nets (PNNs; Celio et al., 1998). PNNs are formed during development (Brückner et al., 2000) in an activity-dependent manner (Dityatev et al., 2007). These structures are composed of hyaluronan, chondroitin sulfate proteoglycans (CSPG), tenascins and link proteins in different ratios and region-specific compositions (Ueno et al., 2018; Fawcett et al., 2019). The appearance of PNNs surrounding PV+ cells marks an abrupt reduction of neuronal plasticity at the end of the critical periods, temporal windows of enhanced neuronal plasticity that occur during the latest stages of development (Hensch, 2005). The PNNs have an important role in the maturation and stabilization of the connectivity of PV + interneurons in critical periods such as those of the visual and auditory systems (Pizzorusso et al., 2002; Cisneros-Franco and de VillersSidani, 2019). The axonal initial segment (AIS) is also a dynamic structure, the length of which is related to neuronal maturation (Gutzmann et al., 2014; Bolós et al., 2019) and also to some pathologic conditions (Kuba et al., 2010; Baalman et al., 2013; Harty et al., 2013). Nevertheless, this parameter has only been studied on excitatory neurons and the impact of the presence of PNNs on the AIS of PV + cells still remains to be explored.

PNNs can be disrupted by the digestion of CSPG, using the enzyme chondroitinase $\mathrm{ABC}(\mathrm{Ch} \mathrm{ABC})$. This treatment promotes neuronal plasticity in different neocortical regions, such as the visual (Pizzorusso et al., 2002) and auditory cortices (Happel et al., 2014). However, the precise mechanisms through which the removal of the PNNs unlocks this plasticity are not clearly understood yet. A recent study in the visual cortex (Lensjø et al., 2017b) has linked the removal of PNNs to a decreased inhibition, suggesting that $\mathrm{ChABC}$ treatment may reset the connectivity to a juvenile-like state. The effects of ChABC have been extensively studied in sensory systems but remain unexplored in high-order areas such as the medial prefrontal cortex (mPFC), whose dysfunction underlies different psychiatric disorders. Moreover, a deficit in the number of PNNs has been described in the PFC of schizophrenic (Mauney et al., 2013) and bipolar patients (Alcaide et al., 2019), supporting the need for further research on the role of PNNs in the etiopathology of psychiatric diseases.

Given the importance of PNNs on the maturation of PV+ interneurons, we analyzed how the presence of PNNs influences the structure, PV expression, and afferent connectivity of these inhibitory neurons in the adult mPFC. Following this line of thought, we also analyzed the impact of the intracerebral injection of ChABC on the efferent and afferent connectivity of PV + cells, especially in the perisomatic region. Finally, we correlated the structural remodeling caused by the enzymatic depletion of PNNs to alterations in the activity of PV + cells, which, in turn, alters $\gamma$ oscillations.

\section{Material and Methods \\ Animals}

Thirty male PV-TdT (C57BL/6-Tg(Pvalb-tdTomato)15Gfng/J, RRID: IMSR_JAX: 027395) three-month-old mice were used in the study. Four animals were used for the descriptive study of $\mathrm{PV}+$ cells. Twenty-six animals were used for the study of ChABC effects on the mPFC: 14 for histologic analysis and 12 for electrophysiological recordings. Animals were housed in groups of two to four in a standard environment (12/12 h light/ dark cycle) and with ad libitum access to food and water. All animal experimentation was conducted in accordance with the Directive 2010/63/EU of the European Parliament and of the Council of 22 September 2010 on the protection of animals used for scientific purposes and was approved by the Committee on Bioethics of the Universitat de València. Every effort was made to minimize the number of animals used and their suffering.

\section{Stereotaxic injection of ChABC}

Mice were anesthetized with isoflurane (4\% for induction, $2 \%$ for maintenance, both in $0.5 \mathrm{ml} \mathrm{O}_{2} / \mathrm{min}$ flow rate). Additionally, dexamethasone $(0.6 \mathrm{mg} / \mathrm{kg})$ was intramuscularly injected to prevent inflammation. Animals were placed in a stereotaxic frame (Narishige), then lidocaine (2\%, Normon) was administered and the skull surface was exposed and dried. Afterwards, trephine holes were drilled in the skull and $1 \mu \mathrm{l}$ of the enzyme ChABC ( $n=13,50 \mathrm{U} / \mathrm{ml}$ in filtered PB; C3667, Sigma-Aldrich) or the control enzyme penicillinase $(n=13,1 \mu \mathrm{g} / \mu \mathrm{l}$ in filtered PB; P0389, Sigma-Aldrich), were bilaterally injected using a Hamilton syringe with a $26-\mathrm{G}$ needle in the following coordinates relative to bregma: anteroposterior +2.00 , mediolateral \pm 0.25 , and dorsoventral -1.00 . The needle was left in position for $2 \mathrm{~min}$ before the injection, and the flow rate during the injection was $100 \mathrm{nl} /$ min. After the injection was completed, the needle was left in place for $5 \mathrm{~min}$ to reduce the reflux of the solution and then slowly withdrawn. Animals were left undisturbed for 2, 4, and $6 \mathrm{~d}$ between the injection and the recording and/or perfusion.

\section{Recording procedure}

Mice were anesthetized with urethane $(1.7 \mathrm{mg} / \mathrm{kg}$, i.p., in sterile saline solution; 94300, Sigma-Aldrich). Ten minutes after the urethane injection, a tracheotomy was performed to minimize respiratory pitfalls during the recording session, following a protocol previously described (Moldestad et al., 2009). Then, the animals were placed in a stereotaxic frame as previously explained and recording electrodes placed at the deep layers of the prelimbic cortex $(\operatorname{PrL})$ : anteroposterior +2.00 , mediolateral +0.25 , and dorsoventral -1.20 from bregma.

Local field potentials (LFPs) were recorded using a formvar insulated stainless steel monopolar macroelectrode $(120 \mu \mathrm{m}$ in diameter, WPI) placed in the PrL. A stainless-steel screw was implanted in the occipital bone as reference. Signals were preamplified $(10 \times$, Grassp551) and amplified $(100 \times$, CIBERTEC Amplifier $)$, bandpass filtered $(0.3$ to $10,000 \mathrm{~Hz})$, digitalized $(10,000 \mathrm{~Hz}$; CED micro 1401 interface), and processed with Spike2 software (Cambridge Electronic Design).

To test the cortical activation, a tail-pinch protocol was used. After 30 min of basal recording, 10 tail stimuli were performed. The tail was stimulated by forceps pressure on the basal zone during $15 \mathrm{~s}$, followed by 3-min resting to recover the basal oscillation pattern; $60 \mathrm{~min}$ after the stimulation protocol started, mice were perfused as explained in the histologic procedures section.

\section{Data analysis}

Data were imported into the MATLAB environment (MathWorks) for the off-line analysis using self-developed code. Recording sessions were divided into brain states of slow oscillations distinguished from $\theta$ periods in response to brief (15 s) sensory-induced (tail pinch) arousal. First, the entire 
A

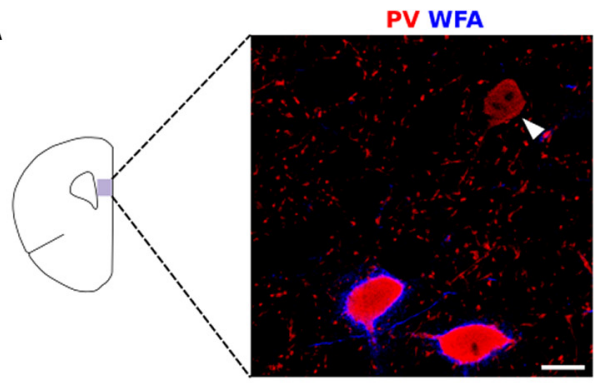

B

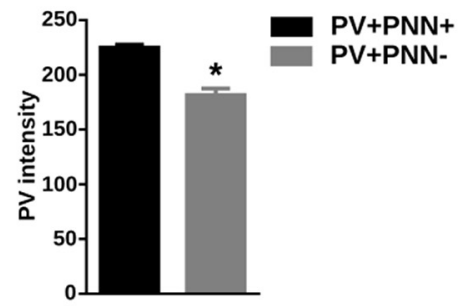

C

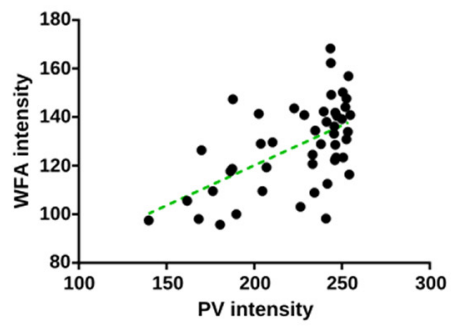

F

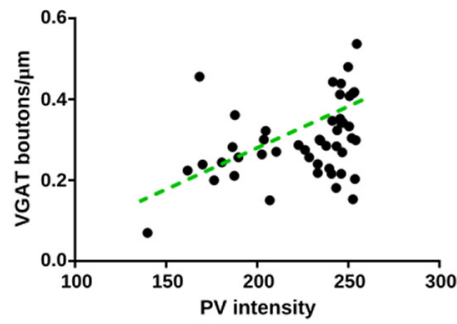

G
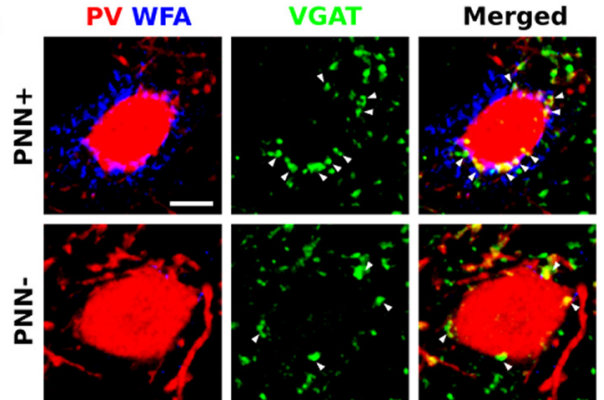

E
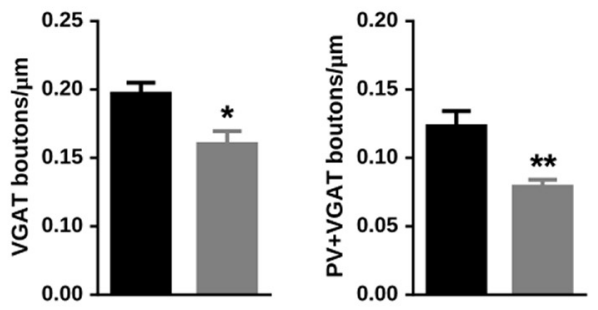

I
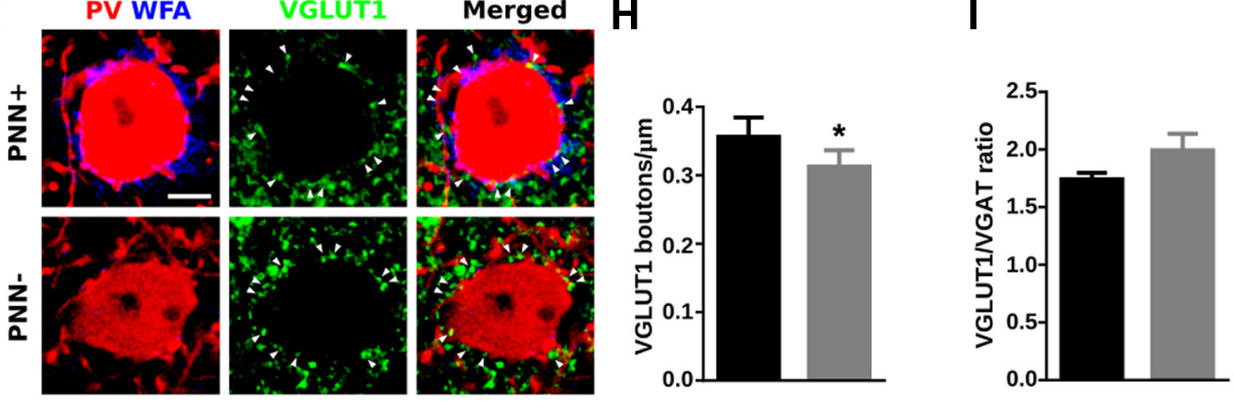

$\mathbf{J}$
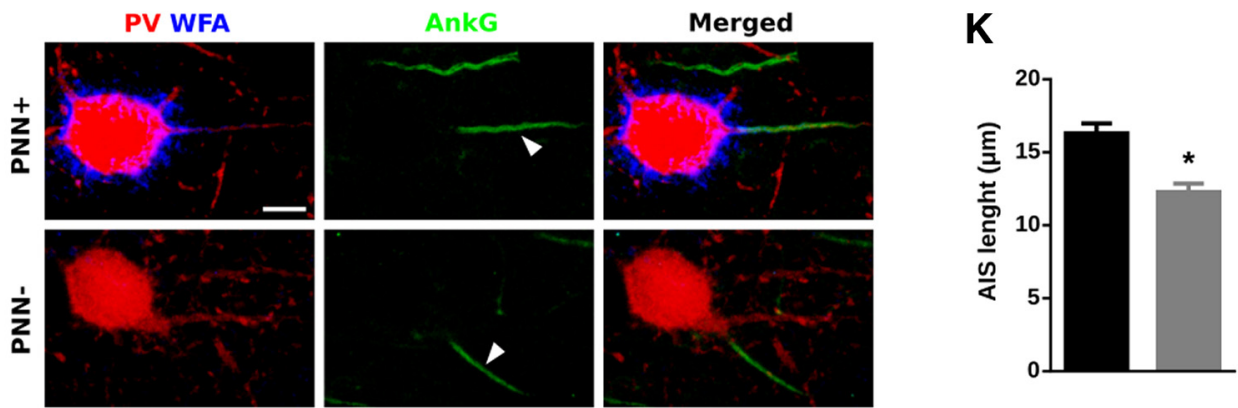

Figure 1. The presence or absence of PNNs surrounding PV+ interneurons influences PV expression in their somata and their perisomatic innervation. $\boldsymbol{A}$, Schematic drawing of a coronal slide, highlighting the area analyzed and a representative immunostaining of the expression of PV and WFA in PrL. The white arrowhead points to a PV+ cell lacking PNN. Scale bar, $10 \mu \mathrm{m}$. $\boldsymbol{B}$, Graph showing the expression of PV in PV+ cells surrounded versus not surrounded by PNNs. C, Distribution graph showing the positive correlation of PV fluorescence intensity and WFA fluorescence intensity. $\boldsymbol{D}$, High-magnification single confocal planes comparing the inhibitory perisomatic innervation on PV + cells depending on the presence or absence of PNNs. White arrowheads point to representative puncta. Scale bar, $4 \mu \mathrm{m}$. $\boldsymbol{E}$, Graphs showing the lower density of perisomatic puncta expressing inhibitory markers on PNN- PV cells. $\boldsymbol{F}$, Distribution graph showing the positive correlation of PV fluorescence intensity and density of inhibitory perisomatic puncta. $\mathbf{G}$, Single confocal planes of VGLUT1-expressing perisomatic puncta on PV + cells. Scale bar, $4 \mu \mathrm{m}$. $\boldsymbol{H}$, Graph showing the density of VGLUT1 + puncta on the perisomatic region of PV + cells. I, Graph comparing the ratio of excitatory/inhibitory perisomatic puncta on PV + cells.J, Representative images of the AIS from PV + cells. White arrowheads point to the AIS. Scale bar, $6 \mu \mathrm{m} . \boldsymbol{K}$, Graph comparing the length of the AIS in PV + cells surrounded versus not surrounded by PNNs. ${ }^{*} p<0.05{ }^{* *} p<0.01$.

recording was bandpass filtered between 0.5 and $250 \mathrm{~Hz}$ (Butterworth filter, low-pass order 4, high-pass order 3) and band-stop filtered $(49.5-50.5,99.5-100.5,149.5-150.5,199.5-$ $200.5 \mathrm{~Hz}$ ) to remove residual $50-\mathrm{Hz}$ power-line contamination and its harmonics.

Spectral analyses were implemented by fast Fourier transform reporting the power distribution in the frequency domain. An estimation of the spectral normalized energy was done using Welch's method (50\% overlapping 4-s Hanning windows with a fast Fourier transform size of $2.56 \mathrm{~Hz}$ and a nonuniform fast Fourier transform value of 1024). The frequency bands defined in the spectral analysis were: $\delta(0.5-3 \mathrm{~Hz})$, low- $\theta(3-6 \mathrm{~Hz})$, high$\theta(6-10 \mathrm{~Hz}), \beta(10-30 \mathrm{~Hz})$, low- $\gamma(30-70 \mathrm{~Hz})$, high- $\gamma(70-$ $100 \mathrm{~Hz})$, and fast $\gamma(>100 \mathrm{~Hz})$. 
Table 1. List of primary and secondary antibodies used

\begin{tabular}{|c|c|c|c|c|c|}
\hline \multicolumn{6}{|c|}{ Primary antibodies } \\
\hline Anti & Host & Isotype & Dilution & Company & Antibody ID \\
\hline AnkG & Mouse & Monoclonal lgG1 & $1: 500$ & Santa Cruz & $A B \_626674$ \\
\hline Camkll & Mouse & Monoclonal lgG1 & $1: 500$ & Abcam & AB_447192 \\
\hline c-Fos & Rabbit & Polyclonal & $1: 500$ & Synaptic Systems & $A B \_2231974$ \\
\hline FosB & Mouse & Monoclonal lgG1 & $1: 500$ & Santa Cruz & AB_783431 \\
\hline PV & Guinea pig & Polyclonal & $1: 2000$ & Synaptic Systems & $A B \_2156476$ \\
\hline PV & Rabbit & Monoclonal & $1: 2000$ & Swant & $A B \_10000344$ \\
\hline SYN & Rabbit & Polyclonal & 1:1000 & Millipore & AB_570874 \\
\hline VGAT & Rabbit & Polyclonal & $1: 500$ & Synaptic Systems & $A B \_887871$ \\
\hline VGLUT1 & Guinea pig & Polyclonal & $1: 2000$ & Millipore & $A B \_2301751$ \\
\hline VGLUT2 & Guinea pig & Polyclonal & $1: 2000$ & Millipore & $A B \_2187552$ \\
\hline
\end{tabular}

Secondary antibodies

\begin{tabular}{|c|c|c|c|c|}
\hline Anti & Host & Label & Dilution & Company \\
\hline Biotin & & A488 & $1: 400$ & Invitrogen \\
\hline Guinea Pig & Donkey & A647 & $1: 400$ & Invitrogen \\
\hline Guinea Pig & Donkey & A405 & $1: 400$ & Invitrogen \\
\hline Mouse lgG1 & Donkey & DL549 & $1: 400$ & Jackson \\
\hline Mouse lgG1 & Donkey & A488 & $1: 400$ & Invitrogen \\
\hline Mouse lgG & Donkey & DL649 & $1: 400$ & Jackson \\
\hline Mouse IgG & Donkey & A555 & $1: 400$ & Molecular Probes \\
\hline Mouse IgM & Donkey & DL649 & $1: 400$ & Jackson \\
\hline Mouse lgM & Donkey & DL549 & $1: 400$ & Jackson \\
\hline Rabbit & Donkey & A405 & $1: 400$ & Invitrogen \\
\hline
\end{tabular}

Cross-frequency interactions between different frequencies of the signals were assessed by the modulation index (MI; Tort et al., 2010), which quantifies the deviation of the phase-amplitude distribution from the uniform distribution through KullbackLeibler divergence. Briefly, raw time series were filtered in the two frequency bands of interest $(\theta$ and $\gamma)$. MI is a normalized measure that reflects how well the instantaneous amplitude of a faster oscillation (amplitude modulated) is phase-locked to an underlying lower cycle (phase modulating). First, the raw signal was filtered at the two frequency ranges under analysis (two-way Butterworth filter with order 2). Then, we applied the Hilbert transform to extract the phase and the instantaneous amplitude (envelope) of both processed signals.

\section{Histologic procedures}

Four days after injection (or $60 \mathrm{~min}$ after the start of recording session for mice destined to electrophysiological analyses), animals were perfused transcardially. Deep anesthesia was induced by sodium pentobarbital $(300 \mathrm{mg} / \mathrm{kg}$, i.p., diluted in distilled water). Animals were perfused transcardially, first with saline and then with paraformaldehyde $4 \%$ in PB $0.1 \mathrm{M}$ for $20 \mathrm{~min}$. After perfusion, brains were extracted and stored in PB $0.1 \mathrm{M}$ with sodium azide $0.05 \%$. Then brains were cut into $50-\mu \mathrm{m}$ sections using a vibratome (VT 1000E, Leica). Sections were collected in six subseries and stored at $4^{\circ} \mathrm{C}$ in $\mathrm{PB} 0.1 \mathrm{~m}$ with sodium azide.

Sections were processed for fluorescence immunohistochemistry as follows. Slices were washed in PBS, then they were incubated for $1 \mathrm{~h}$ in $5 \%$ normal donkey serum (NDS; Invitrogen), $0.2 \%$ Triton X-100 in PBS to block nonspecific binding. After that, sections were incubated for $48 \mathrm{~h}$ at $4^{\circ} \mathrm{C}$ with different primary antibodies cocktails (Table 1 ) diluted in PBS $0.2 \%$ Triton X-100, and a 1:200 diluted biotinylated Wisteria floribunda agglutinin (WFA) for PNNs staining (Sigma-Aldrich L-1516, RRID: AB_2620171). Then, sections were washed and incubated for $2 \mathrm{~h}$ at room temperature with secondary antibody cocktails (Table 1) diluted in PBS $0.2 \%$ Triton X-100. Finally, sections were washed in $0.1 \mathrm{~m} \mathrm{~PB}$, mounted, and coverslipped using fluorescence mounting medium (Dako). For ChABC timeline study in bright field microscopy, sections were washed and incubated for $1 \mathrm{~h}$ with avidin-biotin-peroxidase complex (Vector Laboratories; PK-4000). Then, the sections were incubated for 3 min with 3,3'-diamino-benzidine tetrahydrochloride (Sigma-Aldrich; D5637) and $0.033 \% \mathrm{H}_{2} \mathrm{O}_{2}$ for staining. Sections were finally mounted on slides, dried for $12 \mathrm{~h}$ at room temperature, dehydrated with ascending alcohols, and rinsed in xylene before being coverslipped using Eukitt mounting medium (Sigma-Aldrich; 03989).

\section{Confocal microscopy and image analysis}

Sections were analyzed under a confocal microscope (Leica SPE). Stacks were obtained from Layer V of the PrL at an optimal penetration depth in the tissue (up to $6 \mu \mathrm{m}$ deep). We used a $63 \times$ objective plus $2 \times$ digital zoom for the imaging of perisomatic puncta, neuropil puncta and AIS. A $20 \times$ objective was used for the quantification of cell somata. The acquiring conditions were maintained throughout all the imaging sessions to compare the fluorescence intensity between samples. For the quantification of PV fluorescence intensity, the mean fluorescence intensity was measured in the somata of $100 \mathrm{PV}+$ interneurons manually traced for each experimental condition. For the image analysis of perisomatic puncta, we outlined manually the profile of the cell somata and used a series of custom-made macros in Fiji (Schindelin et al., 2012) as previously described (Guirado et al., 2018). Briefly, after applying subtract background (rolling value $=50)$ and Gaussian blur $(\sigma$ value $=1)$ filters, the original outline was expanded $0.5 \mu \mathrm{m}$ from the cell body surface and the region of interest (ROI) was defined as the area between both outlines. Then, ROI was binarized and puncta quantified after filtering particles for size (included from 0.1 to $1 \mu \mathrm{m}^{2}$ ) and circularity (included from 0.3 to 1 ). For the analysis of neuropil puncta, four small areas of the neuropil $\left(300 \mu \mathrm{m}^{2}\right.$ each) were selected for analysis, to avoid blood vessels and cell somata. Images were processed using previously validated macros (Guirado et al., 2018). To 
compare the effects of PNNs digestion on the density of perisomatic puncta taking into account PV fluorescence intensity, we classified the PV+ cells in two subclasses, using the median value of the PV fluorescence intensity (93.42) as a switch point (the values were uniformly distributed and did not reveal clustering). The AIS length was manually traced after the binarization of confocal planes from AnkyrinG (AnkG)-immunostained sections.

\section{Statistics}

The resulting values were evaluated to apply the adequate statistical analysis. The normality and homoscedasticity of data allowed us to perform parametric analysis on the data. The Pearson correlation coefficient $(r)$ was calculated for correlation analyses. For the comparison in control animals between $\mathrm{PV}+\mathrm{PNN}+$ and $\mathrm{PV}+\mathrm{PNN}-$ cells, we used a paired Student's $t$ test. For ChABC experiments, we used an unpaired Student's $t$ test. All the results were expressed as the mean \pm SEM. All the analysis and graphs were obtained using GraphPad software.

\section{Results}

The presence of PNNs influences the structure, $\mathrm{PV}+$ immunoreactivity, and connectivity of $\mathrm{PV}+$ cells in the mPFC

Considering the important role of PNNs in the maturation of the connectivity of $\mathrm{PV}+$ interneurons, we wondered whether the presence of PNNs, visualized through the histochemical staining of CSPG with WFA, may influence the perisomatic input of these interneurons, PV expression in their somata, and certain structural characteristics. We compared $\mathrm{PV}+$ cells enwrapped by $\mathrm{PNNs}(\mathrm{PV}+\mathrm{PNN}+)$ with those that were not surrounded by this specialized region of the extracellular matrix (PV+ PNN-; Fig. 1). All analyses were performed in PV + cells located in deep layers (V and VI) of the PrL, where these interneurons were more abundant (Fig. 1A). We first studied the intensity of PV immunofluorescence as a readout of the expression of this calcium binding protein, finding $\mathrm{PV}+\mathrm{PNN}+$ cells more intense than $\mathrm{PV}+\mathrm{PNN}-$ cells $(p=0.0310$;

Fig. $1 B$ ). We also analyzed the intensity of WFA fluorescence in the $\mathrm{PV}+\mathrm{PNN}+$ cells and found a significant positive correlation between PV and WFA intensities ( $r=0.5585, p<0.0001$; Fig. $1 C)$. Then, we analyzed the density of perisomatic synaptic puncta onto PV expressing cells. Regarding the puncta expressing markers of inhibitory synapses, we studied PV, VGAT (Fig. 1D) and CB1r (Fig. 2A). We found a significantly lower density of $\mathrm{VGAT}+$ puncta $(p=0.046)$ and of puncta co-expressing VGAT/PV
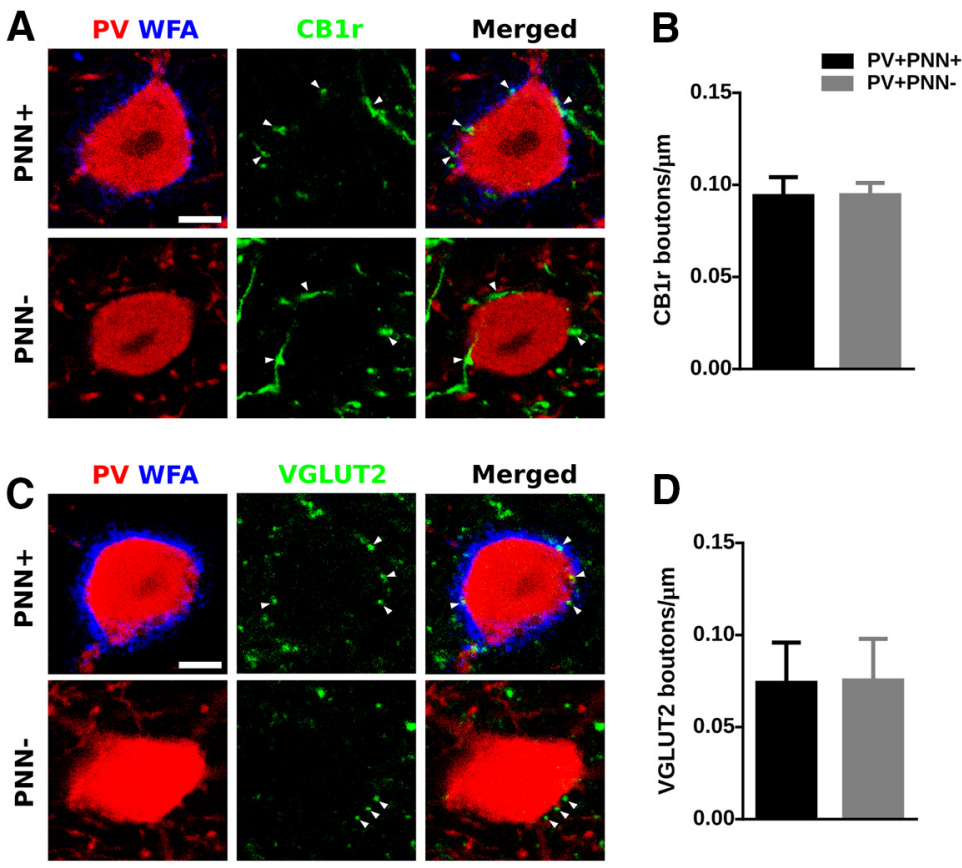

Figure 2. The presence of PNNs does not influence the density of perisomatic puncta coming from $\mathrm{CCK}^{+}$basket cells or extracortical origin on PV+ interneurons. $\boldsymbol{A}$, Representative confocal planes showing CB1r-immunoreactive perisomatic puncta on PV+ cells. Scale bar, $4 \mu \mathrm{m}$. B , Graph comparing the density of CB1r-expressing puncta on PV+ enwrapped by versus lacking PNNs. C, Single confocal planes showing the density of VGLUT2-expressing puncta on the perisomatic region of PV+ cells. Scale bar, $4 \mu \mathrm{m}$. D, Graph showing the density of VGLUT2 + perisomatic puncta on PV + cells, comparing those surrounded by PNNs versus those lacking these extracellular matrix structures.
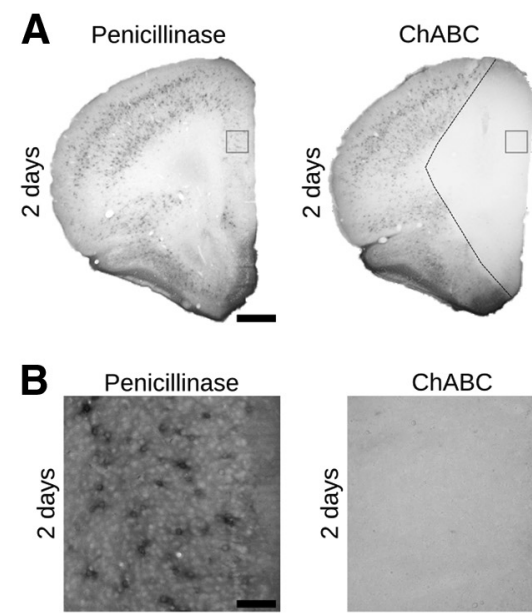

ChABC
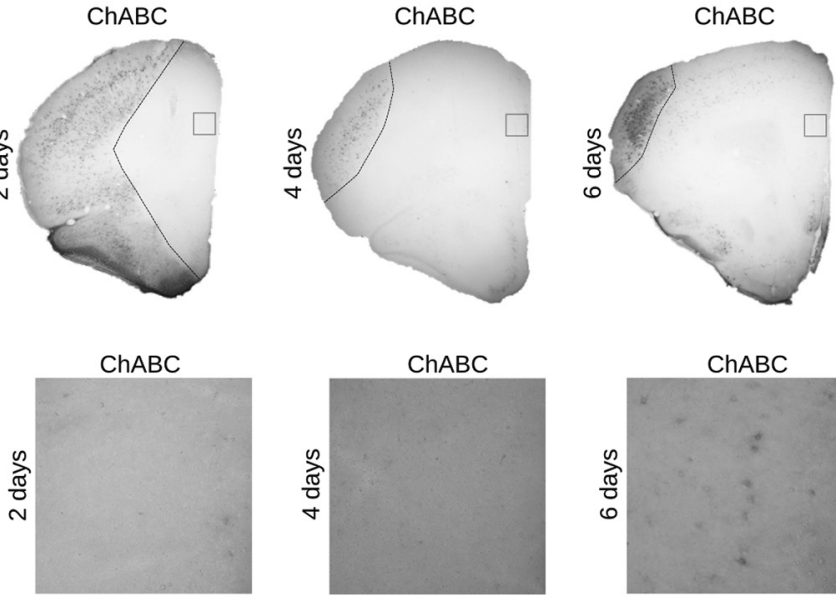

Figure 3. Timeline of PNNs digestion after the intracranial injection of ChABC revealed by WFA labeling. $A$, Microphotographs showing labeled PNNs in the $\mathrm{MPFC}$ of control and ChABC-injected mice. Dashed lines indicate the limits of ChABC effects. Scale bar, $300 \mu \mathrm{m}$. $\boldsymbol{B}$, Insets from the squares in PrL of panel $\boldsymbol{A}$ showing the digestion and the subsequent partial recovery of PNNs. Scale bar, $80 \mu \mathrm{m}$. $(p=0.008$; Fig. $1 E)$ but not of CB1r+ puncta $(p=0.980$; Fig. $2 B)$ in $\mathrm{PV}+\mathrm{PNN}-$ cells. We also found a positive correlation between PV intensity and the density of VGAT + inhibitory perisomatic puncta $(r=0.3864, p=0.0073$; Fig. $1 F)$. Regarding the study of perisomatic puncta expressing markers of excitatory synapses (Fig. 1G), we found a significant decrease in the density of puncta from cortical origin (VGLUT1+; $p=0.031$; Fig. $1 H$ ), but not in that of puncta from extracortical origin (VGLUT2+; $p=0.887$; Fig. $2 C, D$ ) in $\mathrm{PV}+\mathrm{PNN}-$ cells. We then calculated the ratio between excitatory and inhibitory puncta (VGLUT1/VGAT) and we did not find 
A

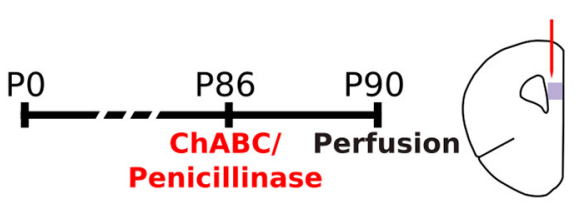

B

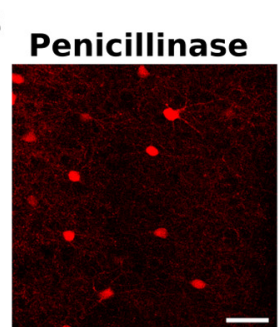

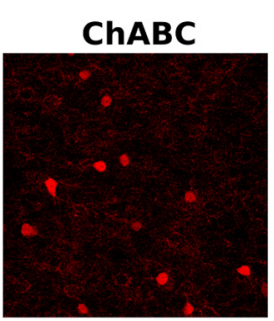

C

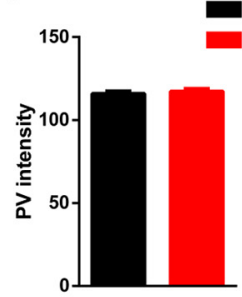

D

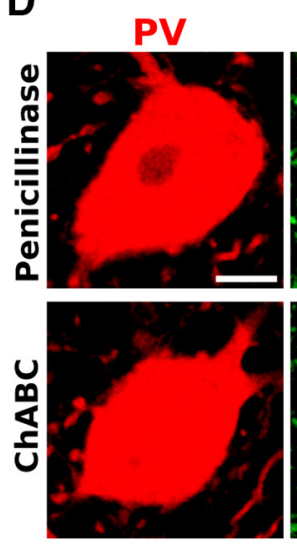

H
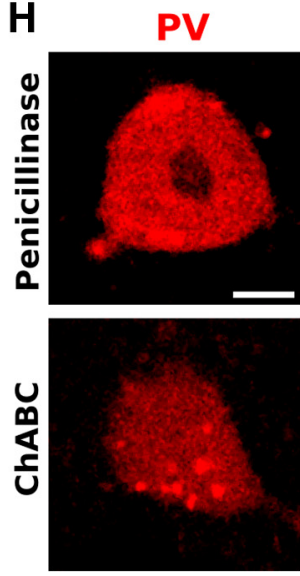

M

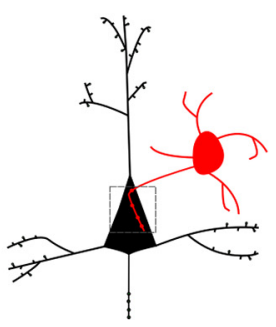

VGLUT1
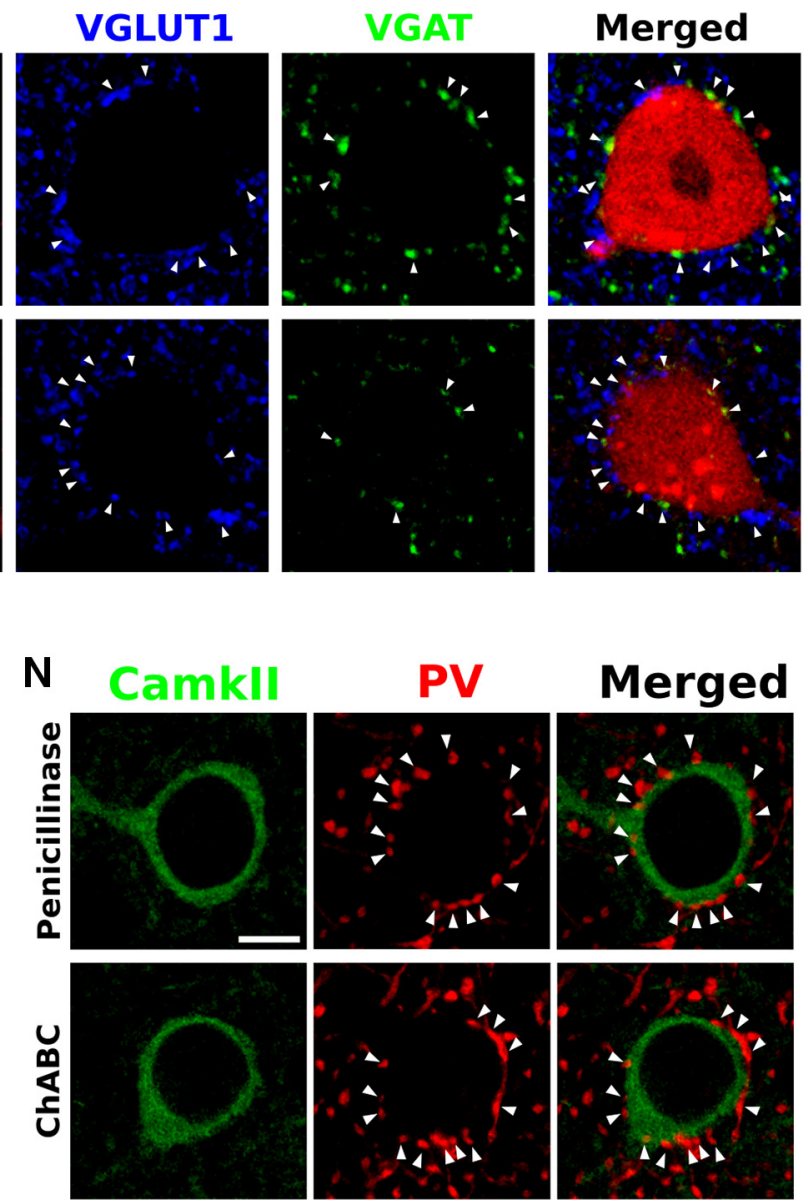

Merged
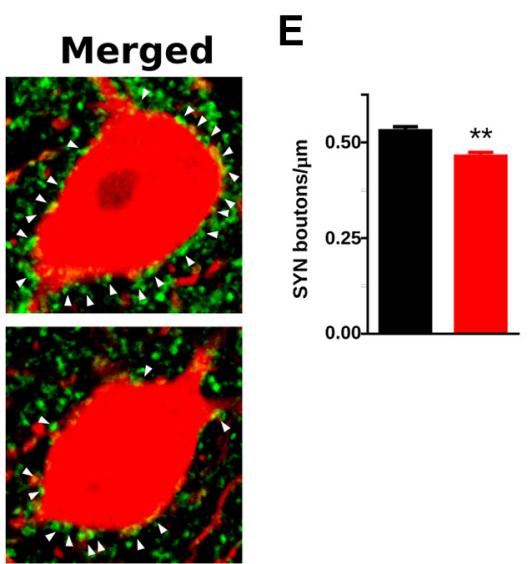

$\mathbf{F}$

Penicillinase ChABC

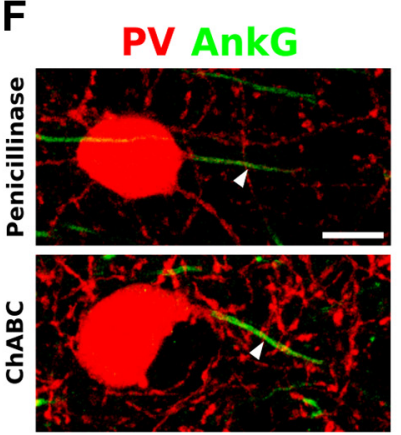

G

I

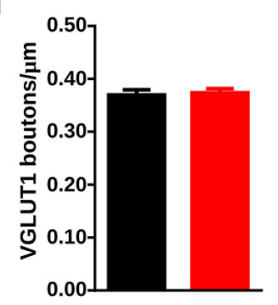

$\mathbf{J}$

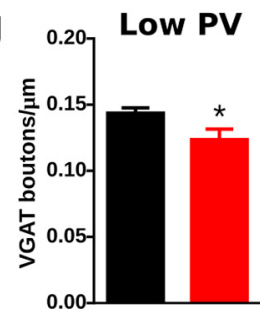

K

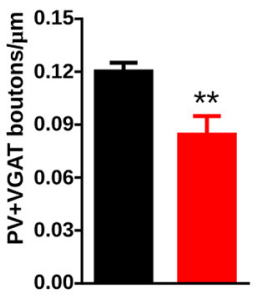

0

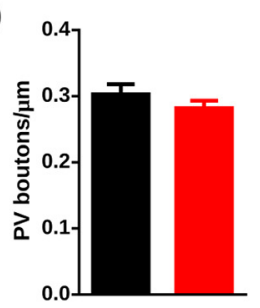

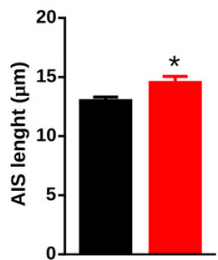
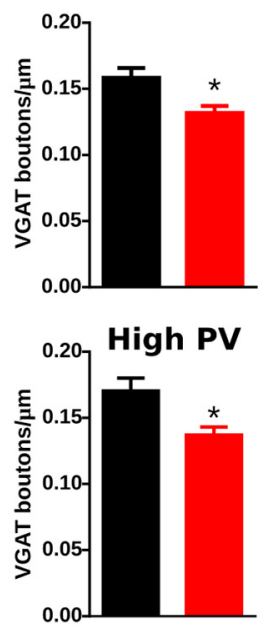

$\mathbf{L}$

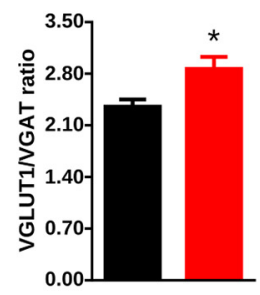

$\mathbf{P}$

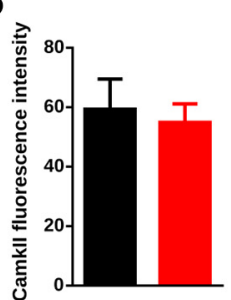

Figure 4. Effects of ChABC on PV expression and perisomatic puncta in the PrL. A, Scheme of experimental timeline and drawing of a coronal slide, highlighting the area of study. $\boldsymbol{B}$, Representative confocal stacks of PV immunostaining in the PrL. C, Graph showing the PV fluorescence intensity in PrL. D, Single confocal planes showing SYN-expressing puncta (white arrowheads) surrounding the soma of a PV+ cell. Scale bar, $5 \mu \mathrm{m}$. $\boldsymbol{E}$, Graphs showing that $\mathrm{ChABC}$ treatment induces a decrease in the density of SYN-expressing puncta on the perisomatic region of PV+ cells. $\boldsymbol{F}$, Confocal reconstruction (three planes) of PV + cells and their AIS. Arrowheads point to AIS stained by AnKG. Scale bar, $8 \mu \mathrm{m}$. $\boldsymbol{G}$, Graph comparing the length of the AIS in $\mathrm{PV}+$ cells after penicillinase and ChABC injection. $\boldsymbol{H}$, Single confocal planes showing the density of excitatory (VGLUT1+) and inhibitory (VGAT+) perisomatic puncta (white arrowheads) on $\mathrm{PV}+$ cells. Scale bar, $5 \mu \mathrm{m}$. I, Graphs showing the ChABC induced decrease in the density of inhibitory but not excitatory puncta on the perisomatic region of PV+ cells. J, Histograms showing 
differences in $\mathrm{PV}+\mathrm{PNN}-$ cells when compared with $\mathrm{PV}+\mathrm{PNN}+$ cells $(p=0.142$; Fig. 1I). As a structural characteristic of the maturational stage, we studied the length of the AIS using AnkG immunohistochemistry (Fig. $1 J$ ) and found that it was significantly shorter in the PV + PNN- cells $(p=0.021$; Fig. $1 K)$. Summarizing, PV-expressing interneurons enwrapped by PNNs displayed a higher expression of PV in their somata and had a higher density of perisomatic inhibitory and excitatory puncta and a longer AIS. We wondered then whether any of these parameters might be also altered when PNNs were digested by $\mathrm{ChABC}$ in the adult $\mathrm{mPFC}$.

\section{ChABC depletes efficiently, but} transiently, the PNNs in the medial PFC We analyzed the temporal progress of the depletion of PNNs after the enzymatic digestion of CSPG by the intracerebral injection of the bacterial enzyme ChABC, comparing it with the effects of the penicillinase injection (Fig. 3A). Two days after the ChABC injection, we found a complete absence of WFA labeling in a large area covering all the $\mathrm{mPFC}$ extension (Fig. $3 B$ ). This depletion was still complete $4 \mathrm{~d}$ after the ChABC injection. Nevertheless, after $6 \mathrm{~d}$, we found a slight recovery of WFA labeling in the perisomatic region of some cells. As our goal was to analyze alterations in the neuronal connectivity before the reappearance of PNNs, we set the histologic and electrophysiological analyses $4 \mathrm{~d}$ after the injection of ChABC.

Effects of ChABC on PV expression and on the density of perisomatic puncta on $\mathrm{PV}+$ cells and pyramidal neurons We studied the effects of ChABC on PV+ cells in the PrL (Fig. $4 A$ ). We quantified the intensity of PV fluorescence (Fig. 4B) and did not find differences in the ChABC-treated animals when compared with controls ( $p=0.694$; Fig. $4 C$ ). Then, we quantified the density of perisomatic synaptic puncta surrounding PV + cells. First, we studied the density of puncta expressing the general presynaptic marker synaptophysin (SYN; Fig. $4 D)$ and we found a significant decrease in ChABC-treated animals ( $p=0.008$; Fig. $4 E$ ). We also studied the effects of PNNs depletion on the AIS length (Fig. $4 F$ ) and found a significant increase as a consequence of ChABC injection ( $p=0.026$; Fig. $4 G$ ). To better understand the origin of this decrease in the perisomatic input, we quantified the density of perisomatic puncta expressing excitatory (VGLUT1) and inhibitory (VGAT) presynaptic markers (Fig. 4H). We found that $\mathrm{ChABC}$ produced a significant decrease in the density of VGAT + puncta $(p=0.0162)$ but not in that of VGLUT1+ puncta

that decrease in inhibitory puncta occurs both in low PV and high PV cells. $\boldsymbol{K}$, Graph showing the significant decrease induced by ChABC on the density of puncta coexpresssing VGAT and $\mathrm{PV}$ in the perisomatic region of PV + cells. $L$, Graphs showing the ratio between the densities of VGLUT1/VGAT-expressing puncta. $\boldsymbol{M}$, Schematic drawing showing the area of analysis highlighted by a gray square. $\boldsymbol{N}$, Single confocal planes showing PV-expressing puncta (white arrowheads) on the perisomatic region of pyramidal neurons. Scale bar, $5 \mu \mathrm{m}$. $\mathbf{0}$, Graph showing the effects of ChABC treatment on the density of PV+ perisomatic puncta on pyramidal neurons. $\boldsymbol{P}$, Graph showing intensity of Camkll fluorescence in the cell body of pyramidal neurons. ${ }^{*} p<0.05,{ }^{* *} p<0.01$.
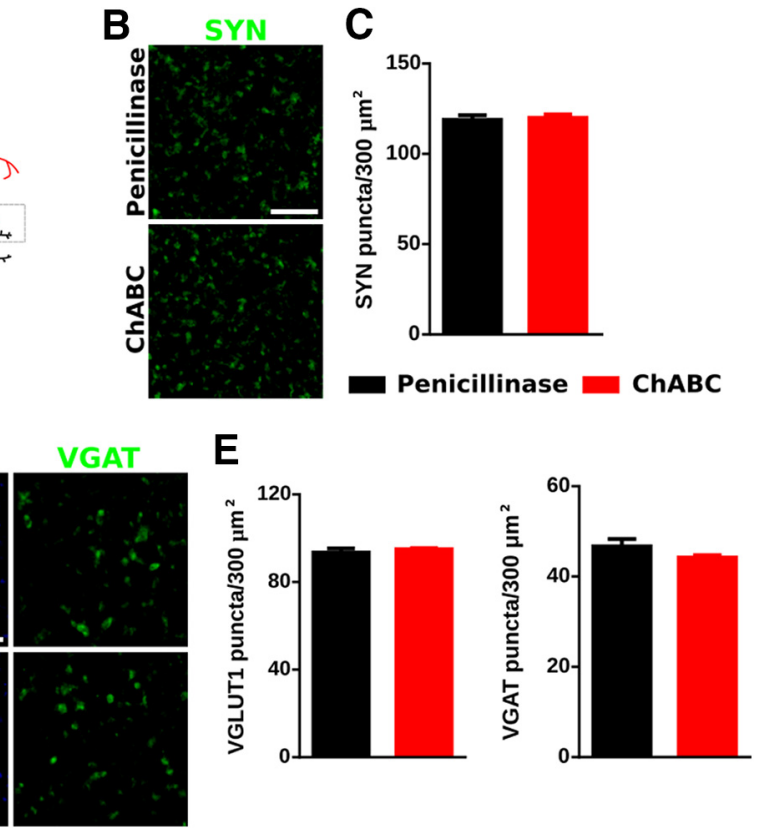

$(p=0.7700$; Fig. 4I). As previous reports have linked the PV expression level to plasticity (Donato et al., 2013, 2015), we wondered whether the effect observed in the perisomatic inhibition was PV expression-dependent. Therefore, we split the PV+ cells into "low PV" and "high PV" attending their PV expression and found that, in both populations, the density of VGAT+ puncta was significantly decreased $(p=0.0464$ for low PV and $p=0.0208$ for high PV; Fig. $4 J)$. We also found that this decreased density of inhibitory puncta most likely corresponded to those coming from $\mathrm{PV}+$ cells, since the density of perisomatic puncta coexpressing PV and VGAT was also decreased ( $p=0.0083$; Fig. $4 K$ ). We analyzed the ratio of VGLUT1/VGAT puncta and found an imbalance toward VGLUT1 in the perisomatic region of PV+ interneurons after ChABC injection ( $p=0.0181$; Fig. $4 L$ ).

Next, we analyzed the synaptic puncta on the perisomatic region of CamkII-expressing pyramidal neurons (Fig. 4M) to detect changes induced by ChABC treatment in the efferent connectivity of PV+ basket interneurons. We analyzed the effect of $\mathrm{ChABC}$ on the density of PV+ puncta (Fig. $4 N$ ) and found that it remained unaltered after the treatment $(p=0.3023$; Fig. 4O). As CamkII expression is involved in neuronal plasticity (Lisman et al., 2012) and could respond to the changes induced by $\mathrm{ChABC}$ treatment, we analyzed its expression through the quantification of its fluorescence intensity in the cell body of pyramidal neuron somata. However, we did not find changes in this parameter $(p=0.7269$; Fig. $4 P)$.

We also analyzed the density of puncta expressing different synaptic markers in the neuropil of the PrL to understand whether the effects observed in the perisomatic area of PV + cells were also extended to other regions (Fig. $5 A$ ). We first studied the density of SYN + puncta and did not find significant changes $(p=0.7573$; Figs. $5 B, C)$. Likewise, the densities of VGLUT1+ and VGAT + puncta were not altered by ChABC $(p=0.482$ and $p=0.221$, respectively; Figs. $5 D, E)$. 
A

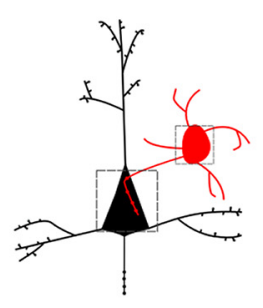

B

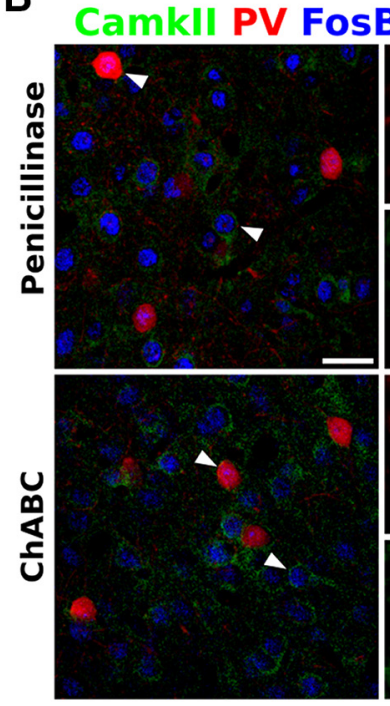

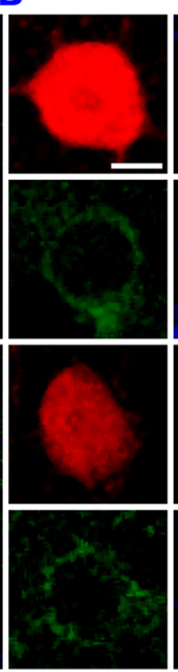

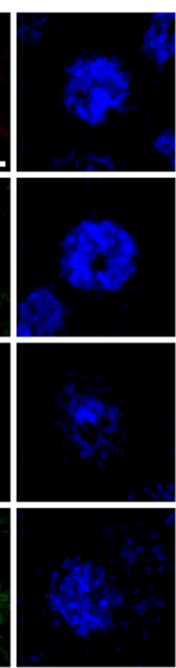

C
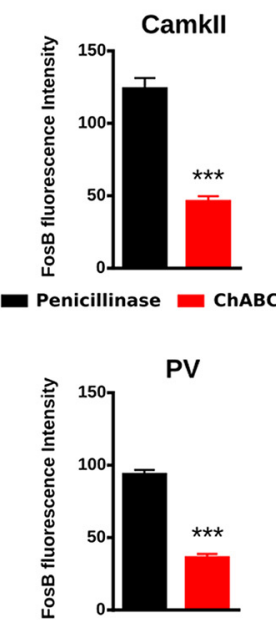

Figure 6. PNNs digestion decreases the fluorescence intensity of FosB in PrL. $\boldsymbol{A}$, Schematic drawing of the area imaged. $\boldsymbol{B}$, Confocal stack (three confocal planes) of PrL of the mPFC showing the expression of PV, Camkll, and FosB. White arrowheads point to higher magnification microphotographs right of each stack showing the FosB expression in PV and Camkll cells. Scale bar, 25 and $3 \mu \mathrm{m}$. C, Graph showing the decrease of the FosB expression in PV cells and Camkll cells in the ChABC animals. ${ }^{* * *} p<0.001$.

\section{$\mathrm{ChABC}$ affects the expression of the basal activity molecule FosB}

We hypothesized that the effects of ChABC on the synaptic puncta described may in turn affect physiology of PrL circuits. Therefore, as an initial approach, we analyzed the expression of the basal activity marker FosB (Nestler, 2015) in PV + cells and pyramidal neurons (Fig. $6 A$ ). The ChABC injection produced a significant decrease of FosB expression in both $\mathrm{PV}+$ cells $(p<0.0001)$ and pyramidal cells $(p<0.0001)$ when compared with penicillinase (Fig. $6 B, C$ ).

\section{ChABC alters $\gamma$ oscillations induced by tail-pinch}

We examined whether the changes described in the density of perisomatic puncta on PV + cells may affect the response of the local neuronal network under $\theta$-elicited stimuli. Four days after ChABC injection, extracellular recordings were made in $\operatorname{PrL}$ under a tailpinch stimulation protocol (Fig. 7A). The tail-pinch was able to evoke $\theta$ rhythmicity, a distinctive oscillation representing characteristic state of the PrL (Fig. $7 B$ ). The power spectra of stimulated epochs revealed a decrease in $\gamma$ oscillations (Fig. 7C,D), both in the high $(p=0.003)$ and fast $\left(p=6 \times 10^{-5}\right)$ ranges (Fig. $\left.7 E\right)$, without significant changes in low $\gamma$ waves $(p=0.65) . \theta-\gamma$ coupling was measured by the MI. No differences were found in control and experimental conditions (Fig. $7 F$ ), indicating the presence of a decreased pattern between $\theta$ and $\gamma$ oscillations $(p=0.109$ for high$\gamma$ and $p=0.6754$ for fast $-\gamma$ ). Finally, we measured the fluorescence intensity of the neuronal activity marker c-Fos in PV+ interneurons and pyramidal neurons $60 \mathrm{~min}$ after the start of the tail-pinch stimulation (Fig. $7 G$ ). We did not find significant differences due to ChABC injection in the PrL $(p=0.1152$ for $\mathrm{PV}+$ interneurons and $p=0.0812$ for pyramidal neurons; Fig. $7 H$ ).

\section{Discussion}

In the present study, we describe differences in the expression of $\mathrm{PV}$ and the density of perisomatic synaptic puncta on PV+ interneurons according to the presence of PNNs. We have found that $\mathrm{PV}+\mathrm{PNN}+$ cells express more $\mathrm{PV}$ and receive a higher density of excitatory and inhibitory perisomatic puncta from other
$\mathrm{PV}+$ cells than $\mathrm{PV}+\mathrm{PNN}-$ cells. Moreover, the digestion of PNNs in PrL produces a reduction of the density of inhibitory puncta, specifically of those expressing PV, on the perisomatic region of $\mathrm{PV}+$ cells. In connection with this reduction, the depletion of PNNs also leads to a reduction in the $\gamma$ activity and the expression of the basal activity marker FosB in the PrL.

To address how the presence of PNNs may affect the connectivity and activity of PV + cells in the PrL, we compared the density of perisomatic puncta expressing different synaptic markers, as well as other molecular and structural characteristics, such as the length of the AIS and the level of expression of PV. We observed that $\mathrm{PV}+\mathrm{PNN}-$ cells display reduced expression levels of $\mathrm{PV}$ when compared with $\mathrm{PV}+\mathrm{PNN}+$ cells. Our results are in agreement with previous work in the hippocampal CA3 (Donato et al., 2013), which proposes a biphasic PV circuitry, with high PV expression cells forming a consolidated circuitry and low PV expression cells sensitive to plastic changes.

Our results show a positive correlation of PV expression and the density of perisomatic inhibitory puncta onto PV + cells. A previous report, focused on hippocampal PV + cells, described that high PV expression cells receive less inhibition and more excitation inputs than low PV expression cells, but in their dendritic compartment (Donato et al., 2013). The comparison of these results suggests different dynamics in the inhibitory input on $\mathrm{PV}+$ cells regarding their PV expression level. It has to be taken also into account that PNNs exclusively enwrap the soma and most proximal segments of dendrites (Celio et al., 1998; Fawcett et al., 2019) and, consequently, should have a more important impact on the perisomatic innervation of PV + cells. Moreover, in the cortex, dendritic inhibition on PV + cells is mainly provided by somatostatin + interneurons, whereas the main source for perisomatic inhibition comes from other PV + cells (Pfeffer et al., 2013). Therefore, we hypothesize that high PV expression cells may receive higher perisomatic inhibition, stabilized by PNNs. By contrast, dendritic inhibition may be decreased in these cells and may be more sensitive to stimuli, such as environmental enrichment (Donato et al., 2013).

We also describe a positive correlation between PNNs and PV expression, in agreement with the described activity-dependent formation of PNNs (Dityatev et al., 2007). Thus, we propose 
A

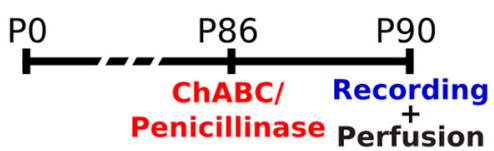

C

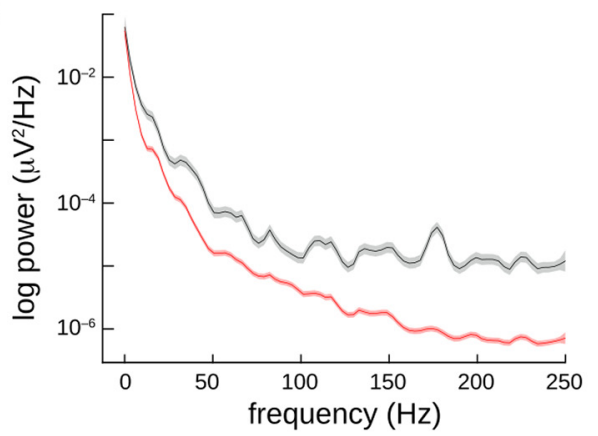

E
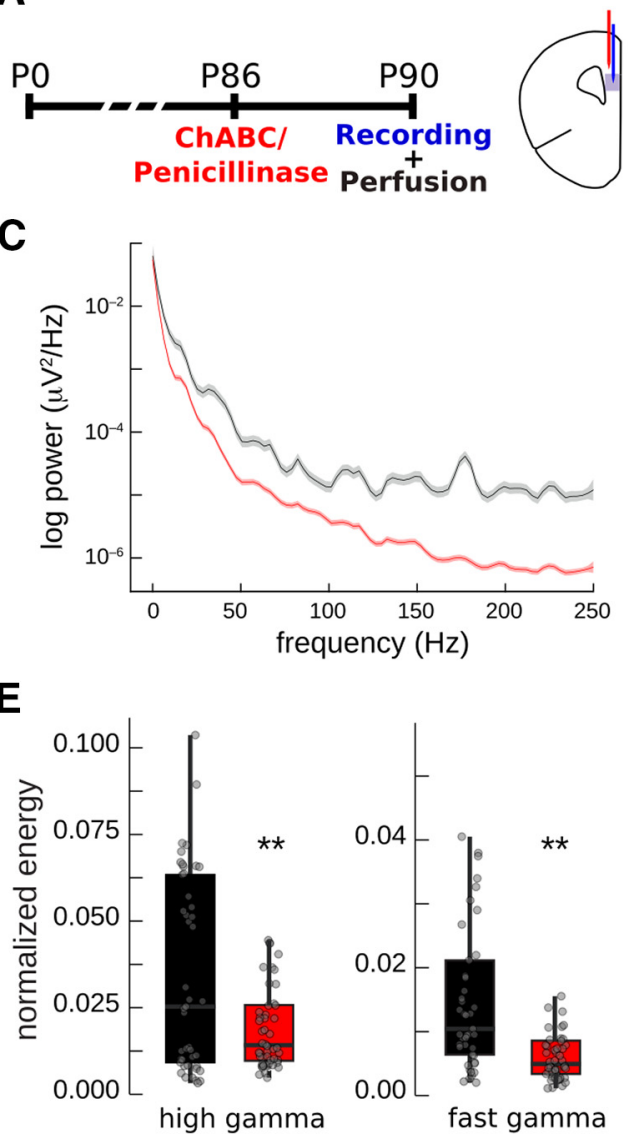

B
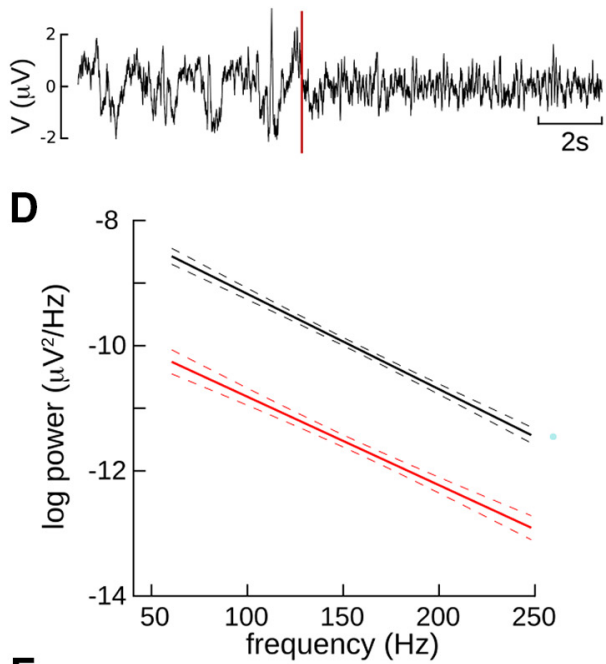

F

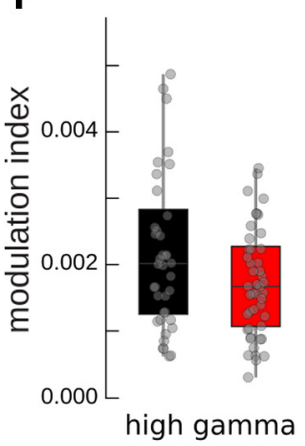

G
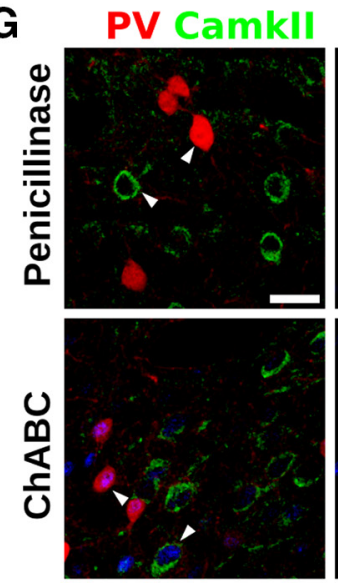
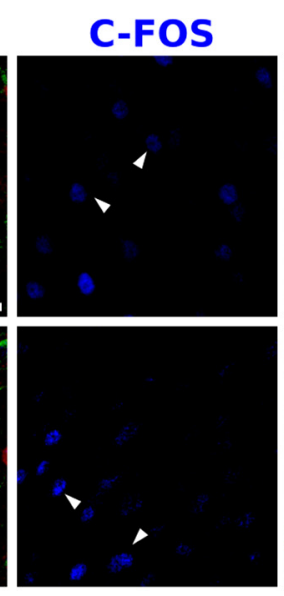

\section{Merged}
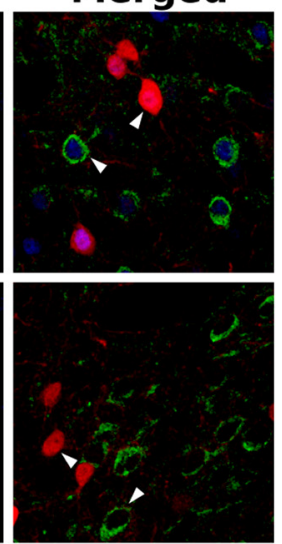

H

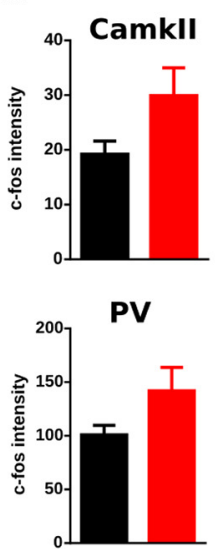

Figure 7. PNNs digestion alters $\gamma$ oscillations in PrL during sensory induced cortical activity. $\boldsymbol{A}$, Scheme of experimental design. $\boldsymbol{B}$, Example of LFP recording in PrL, tail-pinch onset time point is highlighted with a dotted red line. C, Power spectrum of frequencies from 0.5 to $250 \mathrm{~Hz}$ in PrL in a representative case where an average of 10 tail-pinch effects is shown (line, power spectra; shaded area, $95 \%$ confidence interval). D. Fitting of linear models over the grand average of the $\gamma$ power spectra for all experiments. $\boldsymbol{E}$, Box plots of high $\gamma$ and fast $\gamma$ oscillations normalized energy in the PrL. $\boldsymbol{F}, \mathrm{MI}$ of high $\gamma$ and fast $\gamma$ oscillations in the PrL. G, Confocal stack (three planes) on the PrL showing c-Fos expression of PV interneurons and pyramidal neurons after tail-pinch stimulation. White arrowheads point to representative cells. Scale bar, $25 \mu \mathrm{m}$. $\boldsymbol{H}$, Plots showing the fluorescence intensity of $\mathrm{c}-$ Fos in PV interneurons and pyramidal neurons. ${ }^{* *} p<0.01$.

that the establishment of the inhibitory input on the perisomatic region and proximal neurites of PV + cells constitutes a crucial event for the formation of PNNs. Although critical periods are specific for different systems and have a different time lines, the appearance of PNNs coincides with the maturation of $\mathrm{PV}+$ cells and the decrease of plasticity levels, not only in neocortical regions (Pizzorusso et al., 2002; Ueno et al., 2018), but also in the hippocampus (Bukalo et al., 2001) and the amygdala (Gogolla et al., 2009). Therefore, we propose that only PV + cells with a certain degree of innervation from the PV circuitry may develop PNNs and fix their perisomatic connectivity. Supporting this idea, different studies have found an increased cortical plasticity after the induction of a decrease in inhibitory activity, in some cases involving the depletion of PNNs (Harauzov et al., 2010; Toyoizumi et al., 2013; Lensjø et al., 2017b). On the other hand, an experience-dependent accumulation of exogenous OTX2, a 
homeoprotein commonly bound to PNNs and PV + cells in the mPFC (Lee et al., 2017), is necessary for the formation of PNNs and critical period closure in the visual cortex (Sugiyama et al., 2008). Therefore, the formation of PNNs may be the result of a combination of a certain threshold of perisomatic inhibitory activity and OTX2 internalization. We have also analyzed the AIS length and have found that $\mathrm{PV}+\mathrm{PNN}+$ cells had a longer AIS when compared with PV $+\mathrm{PNN}-$ cells. Since the increase in AIS length has been previously related to the maturation of other neuronal types (Sammons et al., 2018; Bolós et al., 2019), our data support the hypothesis of the presence of PNNs as a feature of mature PV + cells, which may have a different role in the PV circuitry from those without PNNs. Taken together, our results suggest the existence of two subpopulations of PV + cells in the mPFC, defined by the presence of PNNs, with differential PV expression and perisomatic connectivity. In order to test whether the depletion of PNNs was able to induce the transformation of most $\mathrm{PV}+$ cells to a phenotype similar to the PV $+\mathrm{PNN}$ - cells in control animals, we tested the effects of ChABC injection in the PrL.

We found that ChABC treatment induces specifically a reduction in the density of inhibitory perisomatic puncta from $\mathrm{PV}+$ cells onto PV + cells, rendering a VGLUT1/VGAT ratio similar to that found in the PV $+\mathrm{PNN}$ - cells in control animals. A recent report has also found a decrease in puncta expressing inhibitory markers after $\mathrm{ChABC}$ treatment in the primary visual cortex (Lensjø et al., 2017a). However, these puncta were quantified in the neuropil and our study focused only on those located in the perisomatic region of PV + cells. Interestingly, we did not find changes in the density of perisomatic puncta on pyramidal neurons, which suggests that $\mathrm{ChABC}$ treatment, despite its impact on the perisomatic input received by $\mathrm{PV}+$ cells, does not induce structural plasticity in the connections that these basket interneurons establish onto pyramidal neurons. However, it is possible that other forms of plasticity, such as the modification of the efficacy of the synapses, may be occurring in these circuits after ChABC treatment, because we have observed alterations in $\gamma$ band oscillations, a rhythm inextricably tied to perisomatic inhibition (Buzsáki and Wang, 2012). It is interesting to note that, unlike Lensjø et al. (2017a), we have not observed changes in the density of puncta expressing synaptic markers in the neuropil of PrL. This discrepancy may be due to the fact that we have analyzed a different neocortical region or to the different survival time after ChABC [7 d in Lensjø et al. (2017a), $4 \mathrm{~d}$ in our study]. The difference may also be due to the type of molecules analyzed, while Lensjø et al. (2017a) analyzed gephyrin, a postsynaptic marker, we measured only presynaptic markers (SYN, VGAT, and VGLUT1).

In order to study whether the ChABC treatment also affected the physiology of neurons in the mPFC, especially that of PV+ basket cells, we conducted electrophysiological studies and analyses of the expression of markers of cellular activity, such as c-Fos and FosB. We used a sensory paradigm to induce a synchronous activated state in the prefrontal neuronal circuitry and, thus, elicit $\theta$ oscillations with a nested $\gamma$ activity (Sohal et al., 2009), a characteristic functional pattern depending on synaptic inhibition onto PV-expressing cells (Buzsáki and Wang, 2012). Apart from external sources of rhythmicity, cortical local networks are prone to synchronize at $\theta$ frequency due to intrinsic oscillations on the substrate of various neuronal types. After ChABC treatment in the PFC, our results showed a decrease of $\gamma$ oscillations, maintaining the $\theta-\gamma$ coupling. Still, the existing $\gamma$ activity maintains its coupling with the $\theta$ waves. Based on these results, we hypothesize that $\theta$ waves are maintained, probably by an external input (Siapas et al., 2005; Sirota et al., 2008), while the local $\gamma$ oscillations reduce their presence by a putative imbalance of the excitatory-inhibitory activity as a consequence of the loss of perisomatic synapses on PV cells. However, we performed our experiments in an associative cortical area by means of temporal stimulus. Thus, we suggest that PNNs digestion leads to a decrease of perisomatic innervation on $\mathrm{PV}+$ cells, which impairs the synchrony of the circuits in which these interneurons are involved, producing a decrease in local $\gamma$ activity. Although we did not find changes after PNNs depletion in the expression of $\mathrm{PV}$, we found a strong reduction in the expression of FosB, not only in $\mathrm{PV}+$ interneurons but also in pyramidal neurons. Since FosB has been linked to long-term activity levels (Nestler, 2015), these data support our electrophysiology results, indicating that the basal activity, not only of PV + cells but also of pyramidal neurons is reduced.

Altogether our results suggest that the disengaging of a portion of $\mathrm{PV}+$ interneurons from the established connectivity patterns is a key mechanism underlying the plasticity observed after PNN removal. This, together with the differences between PV-expressing interneurons surrounded by or lacking PNNs, highlights the importance of this specialized extracellular matrix on PFC function. These results may be relevant for our understanding of the etiopathology of psychiatric disorders, such as schizophrenia or bipolar disorder, in which alterations of prefrontocortical PV + interneurons and PNNs have been described (Mauney et al., 2013; Enwright et al., 2016; Alcaide et al., 2019).

\section{References}

Alcaide J, Guirado R, Crespo C, Blasco-Ibáñez JM, Varea E, Sanjuan J, Nacher J (2019) Alterations of perineuronal nets in the dorsolateral prefrontal cortex of neuropsychiatric patients. Int J Bipolar Disord 7:24.

Baalman KL, Cotton RJ, Rasband SN, Rasband MN (2013) Blast wave exposureimpairs memory and decreases axon initial segment length. J Neurotrauma 30:741-751.

Bolós M, Terreros-Roncal J, Perea JR, Pallas-Bazarra N, Ávila J, LlorensMartín M (2019) Maturation dynamics of the axon initial segment (AIS) of newborn dentate granule cells in young adult C57BL/6J mice. J Neurosci 39:1605-1620.

Brückner G, Grosche J, Schmidt S, Härtig W, Margolis RU, Delpech B, Seidenbecher CI, Czaniera R, Schachner M (2000) Postnatal development of perineuronal nets in wild-type mice and in a mutant deficient in tenascin-R. J Comp Neurol 428:616-629.

Bukalo O, Schachner M, Dityatev A (2001) Modification of extracellular matrix by enzymatic removal of chondroitin sulfate and by lack of tenascin$\mathrm{R}$ differentially affects several forms of synaptic plasticity in the hippocampus. Neuroscience 104:359-369.

Buzsáki G, Wang X-J (2012) Mechanisms of gamma oscillations. Annu Rev Neurosci 35:203-225.

Celio MR, Spreafico R, De Biasi S, Vitellaro-Zuccarello L (1998) Perineuronal nets: past and present. Trends Neurosci 21:510-515.

Cisneros-Franco JM, de Villers-Sidani É (2019) Reactivation of critical period plasticity in adult auditory cortex through chemogenetic silencing of parvalbumin-positive interneurons. Proc Natl Acad Sci USA 116:2632926331.

Dityatev A, Brückner G, Dityateva G, Grosche J, Kleene R, Schachner M (2007) Activity-dependent formation and functions of chondroitin sulfate-rich extracellular matrix of perineuronal nets. Dev Neurobiol 67:570-588.

Donato F, Rompani SB, Caroni P (2013) Parvalbumin-expressing basket-cell network plasticity induced by experience regulates adult learning. Nature 504:272-276.

Donato F, Chowdhury A, Lahr M, Caroni P (2015) Early- and late-born parvalbumin basket cell subpopulations exhibiting distinct regulation and roles in learning. Neuron 85:770-786. 
Enwright JF, Sanapala S, Foglio A, Berry R, Fish KN, Lewis DA (2016) Reduced labeling of parvalbumin neurons and perineuronal nets in the dorsolateral prefrontal cortex of subjects with schizophrenia. Neuropsychopharmacology 41:2206-2214

Fawcett JW, Oohashi T, Pizzorusso T (2019) The roles of perineuronal nets and the perinodal extracellular matrix in neuronal function. Nat Rev Neurosci 20:451-465.

Freund TF, Katona I (2007) Perisomatic inhibition. Neuron 56:33-42.

Gogolla N, Caroni P, Lüthi A, Herry C (2009) Perineuronal nets protect fear memories from erasure. Science 325:1258-1261.

Guirado R, Carceller H, Castillo-Gómez E, Castrén E, Nacher J (2018) Automated analysis of images for molecular quantification in immunohistochemistry. Heliyon 4:e00669.

Gutzmann A, Ergül N, Grossmann R, Schultz C, Wahle P, Engelhardt M (2014) A period of structural plasticity at the axon initial segment in developing visual cortex. Front Neuroanat 8:11.

Happel MFK, Niekisch H, Castiblanco Rivera LL, Ohl FW, Deliano M, Frischknecht R (2014) Enhanced cognitive flexibility in reversal learning induced by removal of the extracellular matrix in auditory cortex. Proc Natl Acad Sci USA 111:2800-2805.

Harauzov A, Spolidoro M, DiCristo G, De Pasquale R, Cancedda L, Pizzorusso T, Viegi A, Berardi N, Maffei L (2010) Reducing intracortical inhibition in the adult visual cortex promotes ocular dominance plasticity. J Neurosci 30:361-371.

Harty RC, Kim TH, Thomas EA, Cardamone L, Jones NC, Petrou S, Wimmer VC (2013) Axon initial segment structural plasticity in animal models of genetic andacquired epilepsy. Epilepsy Res 105:272-279.

Hensch TK (2005) Critical period plasticity in local cortical circuits. Nat Rev Neurosci 6:877-888.

Kuba H, Oichi Y, Ohmori H (2010) Presynaptic activity regulates $\mathrm{Na}(+)$ channel distribution at the axon initial segment. Nature 465:1075-1078.

Kubota Y (2014) Untangling GABAergic wiring in the cortical microcircuit. Curr Opin Neurobiol 26:7-14.

Lee HHC, Bernard C, Ye Z, Acampora D, Simeone A, Prochiantz A, Di Nardo AA, Hensch TK (2017) Genetic Otx2 mis-localization delays critical period plasticity across brain regions. Mol Psychiatry 22:680-688.

Lensjø KK, Christensen AC, Tennøe S, Fyhn M, Hafting T (2017a) Differential expression and cell-type specificity of perineuronal nets in hippocampus, medial entorhinal cortex, and visual cortex examined in the rat and mouse. eNeuro 4: ENEURO.0379-16.2017.

Lensjø KK, Lepperød ME, Dick G, Hafting T, Fyhn M (2017b) Removal of perineuronal nets unlocks juvenile plasticity through network mechanisms of decreased inhibition and increased gamma activity. J Neurosci 37:1269-1283.

Lisman J, Yasuda R, Raghavachari S (2012) Mechanisms of CaMKII action in long-term potentiation. Nat Rev Neurosci 13:169-182.
Mauney SA, Athanas KM, Pantazopoulos H, Shaskan N, Passeri E, Berretta S, Woo T-U (2013) Developmental pattern of perineuronal nets in the human prefrontal cortex and their deficit in schizophrenia. Biol Psychiatry 74:427-435.

Moldestad O, Karlsen P, Molden S, Storm JF (2009) Tracheotomy improves experiment success rate in mice during urethane anesthesia and stereotaxic surgery. J Neurosci Methods 176:57-62.

Nestler EJ (2015) $\Delta$ FosB: a transcriptional regulator of stress and antidepressant responses. Eur J Pharmacol 753:66-72.

Pfeffer CK, Xue M, He M, Huang ZJ, Scanziani M (2013) Inhibition of inhibition in visual cortex: the logic of connections between molecularly distinct interneurons. Nat Neurosci 16:1068-1076.

Pizzorusso T, Medini P, Berardi N, Chierzi S, Fawcett JW, Maffei L (2002) Reactivation of ocular dominance plasticity in the adult visual cortex. Science 298:1248-1251.

Sammons RP, Clopath C, Barnes SJ (2018) Size-dependent axonal bouton dynamics following visual deprivation in vivo. Cell Rep 22:576-584.

Schindelin J, Arganda-Carreras I, Frise E, Kaynig V, Longair M, Pietzsch T, Preibisch S, Rueden C, Saalfeld S, Schmid B, Tinevez JY, White DJ, Hartenstein V, Eliceiri K, Tomancak P, Cardona A (2012) Fiji: an opensource platform for biological-image analysis. Nat Methods 9:676-682.

Siapas AG, Lubenov EV, Wilson MA (2005) Prefrontal phase locking to hippocampal theta oscillations. Neuron 46:141-151.

Sirota A, Montgomery S, Fujisawa S, Isomura Y, Zugaro M, Buzsáki G (2008) Entrainment of neocortical neurons and gamma oscillations by the hippocampal theta rhythm. Neuron 60:683-697.

Sohal VS, Zhang F, Yizhar O, Deisseroth K (2009) Parvalbumin neurons and gamma rhythms enhance cortical circuit performance. Nature 459:698702.

Sugiyama S, Di Nardo AA, Aizawa S, Matsuo I, Volovitch M, Prochiantz A, Hensch TK (2008) Experience-dependent transfer of Otx2 homeoprotein into the visual cortex activates postnatal plasticity. Cell 134:508-520.

Tort ABL, Komorowski R, Eichenbaum H, Kopell N (2010) Measuring phase-amplitude coupling between neuronal oscillations of different frequencies. J Neurophysiol 104:1195-1210.

Toyoizumi T, Miyamoto H, Yazaki-Sugiyama Y, Atapour N, Hensch TK, Miller KD (2013) A theory of the transition to critical period plasticity: inhibition selectively suppresses spontaneous activity. Neuron 80:51-63.

Tremblay R, Lee S, Rudy B (2016) GABAergic interneurons in the neocortex: from cellular properties to circuits. Neuron 91:260-292.

Ueno H, Takao K, Suemitsu S, Murakami S, Kitamura N, Wani K, Okamoto M, Aoki S, Ishihara T (2018) Age-dependent and region-specific alteration of parvalbumin neurons and perineuronal nets in the mouse cerebral cortex. Neurochem Int 112:59-70. 